\title{
Reaction models in nuclear astrophysics
}

\author{
Pierre Descouvemont
}

Physique Nucléaire Théorique et Physique Mathématique, C.P. 229, Université Libre de Bruxelles (ULB), B 1050 Brussels, Belgium

\begin{abstract}
We present different reaction models commonly used in nuclear astrophysics, in particular for the nucleosynthesis of light elements. Pioneering works were performed within the potential model, where the internal structure of the colliding nuclei is completely ignored. Significant advances in microscopic cluster models provided the first microscopic description of the ${ }^{3} \mathrm{He}(\alpha, \gamma){ }^{7}$ Be reaction more than thirty years ago. In this approach, the calculations are based on an effective nucleon-nucleon interaction, but the cluster approximation should be made to simplify the calculations. Nowadays, modern microscopic calculations are able to go beyond the cluster approximation, and aim at finding exact solutions of the Schrödinger equation with realistic nucleon-nucleon interactions. We discuss recent examples on the $d+d$ reactions at low energies.
\end{abstract}

\section{Introduction}

Nuclear reactions determine the nucleosynthesis in stars, and produce the energy released to compensate their gravitational contraction [1]. Stellar models are in general based on large reaction networks, involving many reaction rates. In the Big Bang nucleosynthesis, only a few reactions are important, producing elements up to $A=8$. In stellar nucleosynthesis, the reaction networks depend on the physical conditions of the star (mass, temperature, density, etc.). At low temperatures, the stellar evolution is mainly determined by the pp chain and by the CNO cycle. Both processes convert 
hydrogen in Helium. Advanced stages of stellar evolution involve He burning, followed by reactions involving heavier elements. At high temperature, neutron capture ( $s$ and $p$ processes), as well as explosive burning determine the star evolution.

The calculation of the reaction rates relies on the cross sections [2]. There are in general two main problems in nuclear astrophysics: (i) the stellar energies being much smaller than the Coulomb barrier, the relevant cross sections between charged particles are too small to be measured in the laboratory; (ii) explosive burning involves short-live nuclei which, even if they can be produced with modern technologies, are available with weak intensities only. Consequently a theoretical support is necessary, either to extrapolate the cross sections down to astrophysical energies, or to predict unknown cross sections.

Theoretical models used in nuclear astrophysics can be roughly classified in three categories $[3,4]$ :

(i) Models involving adjustable parameters, such as the $R$-matrix method $[5,6]$; parameters are fitted to the available experimental data and the cross sections are extrapolated down to astrophysical energies. These fitting procedures of course require the knowledge of data, which are sometimes too scarce for a reliable extrapolation.

(ii) "Non fitting" models, where the cross sections are determined from the wave functions of the system. The potential model [7], the Distorted Wave Born Approximation (DWBA) [8], and microscopic models [9] are, in principle, independent of experimental data. More realistically, these models depend on some physical parameters, such as a nucleus-nucleus or a nucleon-nucleon interaction which can be reasonably determined from experiment only.

(iii) Models $(i)$ and (ii) can be used for low level-density nuclei only. This condition is fulfilled in most of the reactions involving light nuclei $(A \leq 20)$. However when the level density near threshold is large (i.e. more than a few levels per $\mathrm{MeV}$ ), statistical models, using averaged optical transmission coefficients, are in general more suitable [10]. In some specific applications, shell-model theories can provide the gamma widths of relevant states.

\section{Theoretical models}

As mentioned before, reaction models are essential in nuclear astrophysics. Many models have been used to describe low-energy reactions. Here we give a brief overview of various approaches, commonly used in the field. 


\subsection{The potential model}

Solving the Schrödinger equation associated with a many-body system is a difficult problem, which does not have an exact solution when the nucleon number is larger than three. The potential model is fairly simple to use, and has been applied to many reactions in low-energy nuclear physics $[7,11]$. The basic assumptions of the potential model are: $(i)$ the nucleon-nucleon interaction is replaced by a nucleus-nucleus force $V(\rho)$, which depends on the relative coordinate $\rho$ only; (ii) the wave functions of the unified nucleus can be described by a cluster structure with $A_{1}+A_{2}$ nucleons; (iii) the internal structure of the nuclei does not play any role. Since we are dealing with low energies, the potential is in general real. The extension to higher energies, which requires complex potentials to simulate absorption channels, is known as the optical model. A generalization to coupled-channel problems is also possible, but seldom used in nuclear astrophysics.

The radial function $g_{\ell J}^{J \pi}(\rho)$ for bound and scattering states is deduced from the equation

$$
\left(-\frac{\hbar^{2}}{2 \mu m_{N}}\left(\frac{d^{2}}{d r^{2}}-\frac{\ell(\ell+1)}{r^{2}}\right)+V(\rho)\right) g_{\ell J}^{J \pi}(\rho)=E g_{\ell J}^{J \pi}(\rho),
$$

where $E$ is the relative energy ( $E>0$ for scattering states and $E<0$ for bound states). Let us notice that the potential may depend on $\ell$ and $J$. In nuclear physics, the nucleus-nucleus potential involves a Coulomb term $V_{C}(\rho)$ and a nuclear term $V_{N}(\rho)$. According to the application, the choice of the nuclear contribution is guided by experimental constraints. In radiativecapture calculations it is crucial to reproduce the final-state energy. If phase shifts are available, they can be used to determine the initial potential.

In this simple model, the capture cross sections are deduced from integrals involving scattering functions $g_{\ell_{i} I}^{J_{i} \pi_{i}}(\rho)$ at energy $E$, and bound-state wave functions $g_{\ell_{f} I}^{J_{f} \pi_{f}}(\rho)$ (see Ref. [11] for detail).

\subsection{The phenomenological $R$-matrix method}

The $R$-matrix method is well known in atomic and nuclear physics [6]. The basis idea is to divide the space in two regions: the internal region (with radius $a$ ), where the nuclear force is important, and the external region, where the interaction between the nuclei is governed by the Coulomb force only. Although the $R$-matrix parameters do depend on the channel radius $a$, the sensitivity of the cross section with respect to its choice is quite weak. In the 
$R$-matrix method, the energy dependence of the cross sections is obtained from Coulomb functions, as expected from the Schrödinger equation.

The physics of the internal region is determined by a number $N$ of poles, which are characterized by energy $E_{\lambda}$ and reduced widths $\gamma_{\lambda i}$. In a multichannel problem, the $R$-matrix at energy $E$ is defined as

$$
R_{i f}(E)=\sum_{\lambda=1}^{N} \frac{\gamma_{\lambda i} \gamma_{\lambda f}}{E_{\lambda}-E},
$$

which must be given for each partial wave $J$ (not written for the sake of clarity). Indices $i$ and $f$ refer to the initial and final channels. The pole properties are associated with the physical energy and width of resonances, but not strictly equal. This is known as the difference between "formal" and "observed" parameters, deduced from experiment. In a general case, involving more than one pole, the link between those two sets is not straightforward (see Refs. [12,13] for a general formulation of this problem). The collision matrix, and therefore the cross sections, are directly determined from the $R$ matrices in the different partial waves (see Refs. $[5,6]$ ).

The $R$-matrix method can be applied to transfer as well as to capture reactions. It is usually used to investigate resonant reactions but is also suited to describe non-resonant processes. In the latter case, the non-resonant behavior is simulated by a high-energy pole, referred to as the background contribution, which makes the $R$-matrix nearly energy independent.

\subsection{Microscopic cluster theories}

Microscopic models are based on fundamental principles of quantum mechanics, such as the treatment of all nucleons, with exact antisymmetrization of the wave functions. Neglecting three-body forces, the Hamiltonian of a $A$-nucleon system is written as

$$
H=\sum_{i=1}^{A} T_{i}+\sum_{i<j=1}^{A} V_{i j},
$$

where $T_{i}$ is the kinetic energy and $V_{i j}$ a nucleon-nucleon interaction [9].

The Schrödinger equation associated with this Hamiltonian can not be solved exactly when $A>3$. For very light systems $(A \sim 4-5)$ efficient methods [14] exist, even for continuum states [15]. However most reactions relevant in nuclear astrophysics involve heavier nuclei essentially with nucleon or $\alpha$ projectiles. Recent developments of ab initio models (see for 
example Refs. [16-18]) are quite successful for spectroscopic properties of low-lying states. These models make use of realistic interactions, including three-body forces, and fitted on many properties of the nucleon-nucleon system. Recent works succeeded in computing the ${ }^{3} \mathrm{He}(\alpha, \gamma){ }^{7} \mathrm{Be}[17]$ and ${ }^{2} \mathrm{H}(d, \gamma){ }^{4} \mathrm{He}[19]$ cross sections from realistic interactions. However, a consistent description of bound and scattering states of an $A$-body problem remains a very difficult task [15], in particular for transfer reactions.

In the cluster approximation, it is assumed that the nucleons are grouped in clusters $[9,20]$. The internal wave functions of the clusters are denoted as $\phi_{i}^{I_{i} \pi_{i} \nu_{i}}\left(\boldsymbol{\xi}_{\boldsymbol{i}}\right)$, where $I_{i}$ and $\pi_{i}$ are the spin and parity of cluster $i$, and $\boldsymbol{\xi}_{\boldsymbol{i}}$ represents a set of their internal coordinates. In a two-cluster system, a channel function is defined as

$$
\varphi_{\ell I}^{J M \pi}\left(\Omega_{\rho}, \boldsymbol{\xi}_{\mathbf{1}}, \boldsymbol{\xi}_{\mathbf{2}}\right)=\left[Y_{\ell}\left(\Omega_{\rho}\right) \otimes\left[\phi_{1}^{I_{1} \pi_{1}}\left(\boldsymbol{\xi}_{\mathbf{1}}\right) \otimes \phi_{2}^{I_{2} \pi_{2}}\left(\boldsymbol{\xi}_{\mathbf{2}}\right)\right]^{I}\right]^{J M},
$$

where different quantum numbers show up: the channel spin $I$, the relative angular momentum $\ell$, the total spin $J$ and the total parity $\pi=\pi_{1} \pi_{2}(-)^{\ell}$.

The total wave function of the $A$-nucleon system is written as

$$
\Psi^{J M \pi}=\sum_{\alpha \ell I} \Psi_{\alpha \ell I}^{J M \pi}=\sum_{\alpha \ell I} \mathcal{A} g_{\alpha \ell I}^{J \pi}(\rho) \varphi_{\alpha \ell I}^{J M \pi}\left(\Omega_{\rho}, \boldsymbol{\xi}_{\mathbf{1}}, \boldsymbol{\xi}_{\mathbf{2}}\right),
$$

which corresponds to the Resonating Group (RGM) definition [20,21]. Index $\alpha$ refers to different two-cluster arrangements, and $\mathcal{A}$ is the antisymetrization operator. In most applications, the internal cluster wave functions $\phi_{i}^{I_{i} \pi_{i} \nu_{i}}$ are defined in the shell model. Accordingly, the nucleon-nucleon interaction must be adapted to this choice, which leads to effective forces, such as the Volkov [22] or the Minnesota [23] interactions. The relative wave functions $g_{\alpha \ell I}^{J \pi}(\rho)$ are to be determined from the Schrödinger equation, which is transformed into a integro-differential equation involving a non-local potential [21]. In most applications, this relative function is expanded over Gaussian functions [9, 24], which corresponds to the Generator Coordinate Method (GCM).

The main advantage of cluster models with respect to other microscopic theories is their ability to deal with reactions, as well as with nuclear spectroscopy. The first applications were done for reactions involving light nuclei, such as d, ${ }^{3} \mathrm{He}$ or $\alpha$ particles $[25,26]$. More recently, much work has been devoted to the improvement of the internal wave functions: multicluster descriptions [27], large-basis shell model extensions [28], or monopolar distortion [29]. 


\subsection{Ab-initio models: the ${ }^{2} \mathbf{H}(d, \gamma){ }^{4} \mathbf{H e}, \quad{ }^{2} \mathbf{H}(d, p)^{3} \mathbf{H}$ and ${ }^{2} \mathbf{H}(d, n){ }^{3} \mathbf{H e}$ cross sections}

The knowledge of the reaction cross sections at astrophysical energies is of great interest not only for establishing imprints of the properties of nuclei in the universe but also for understanding an interplay between the structure and reactions of these nuclei based on a nucleon-nucleon $(N N)$ interaction. Aside from the astrophysical interest, the ${ }^{2} \mathrm{H}(d, \gamma){ }^{4} \mathrm{He}$ capture reaction is extremely important from the nuclear physics viewpoint because its cross section at low energies (below $0.3 \mathrm{MeV}$ ) is expected to be dominated by $D$-wave components in the $\alpha$ particle. Hence it should be very sensitive to the tensor force in the $N N$ interaction [30].

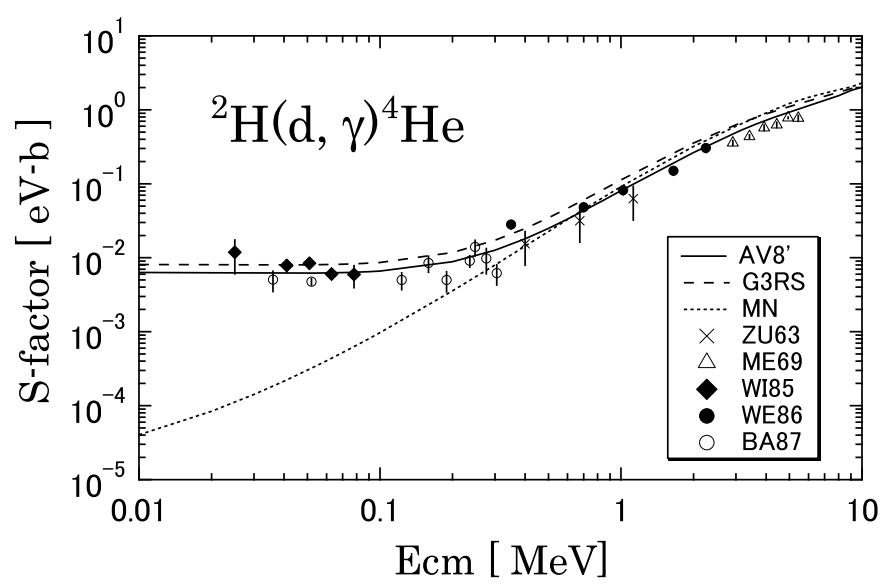

Figure 1: The astrophysical $S$-factor of the ${ }^{2} \mathrm{H}(d, \gamma){ }^{4} \mathrm{He}$ reaction (taken from Ref. [19]). Results calculated with the realistic (AV8', G3RS) and the effective (MN) potentials are compared to experiment (see Ref. [34]).

We have applied an ab initio model to study the phase shifts of the $p+{ }^{3} \mathrm{He}$ [31] and $d+d, p+{ }^{3} \mathrm{H}, n+{ }^{3} \mathrm{He}$ [19] systems. For the two-body $N N$ interaction $V_{i j}$, we use two different realistic potentials, $\mathrm{AV}^{\prime}$ [32] and G3RS [33], that consist of central, tensor, and spin-orbit components. Because our main aim is to clarify the role of the tensor force, it is useful to compare results obtained with the realistic interactions with that of an effective $N N$ interaction that contains no tensor force. Using such effective interaction is reasonable because only $s$-shell nuclei participate to the reactions. We adopt the MN central potential [23] with a standard value for the admixture parameter $u=1$.

Figure 1 displays the calculated astrophysical $S$-factor for the ${ }^{2} \mathrm{H}(d, \gamma)^{4} \mathrm{He}$ 
reaction. Results with both $\mathrm{AV}^{\prime}$ (solid line) and G3RS (dashed line) potentials reproduce very well the experimental data, especially its flat behavior at low energies (typical of an initial $s$ wave), whereas the MN potential (dotted curve) shows a rapidly decreasing pattern as $E_{\mathrm{cm}}$ decreases. Without the tensor force, we cannot reproduce the ${ }^{2} \mathrm{H}(d, \gamma){ }^{4} \mathrm{He}$ astrophysical $S$-factor below $0.3 \mathrm{MeV}$.

Similar conclusions can be drawn for the ${ }^{2} \mathrm{H}(d, p){ }^{3} \mathrm{H}$ and ${ }^{2} \mathrm{H}(d, n)^{3} \mathrm{He}$ transfer reactions at low energies. The $S$ factors are presented in Fig. 2. They mainly occur from the transitions of the $d+d{ }^{5} S_{2}$ channel to the $D$ wave continuum of $p+{ }^{3} \mathrm{H}$ or $n+{ }^{3} \mathrm{He}$, which is also due to the tensor force. Without the tensor force, these cross sections cannot be reproduced.

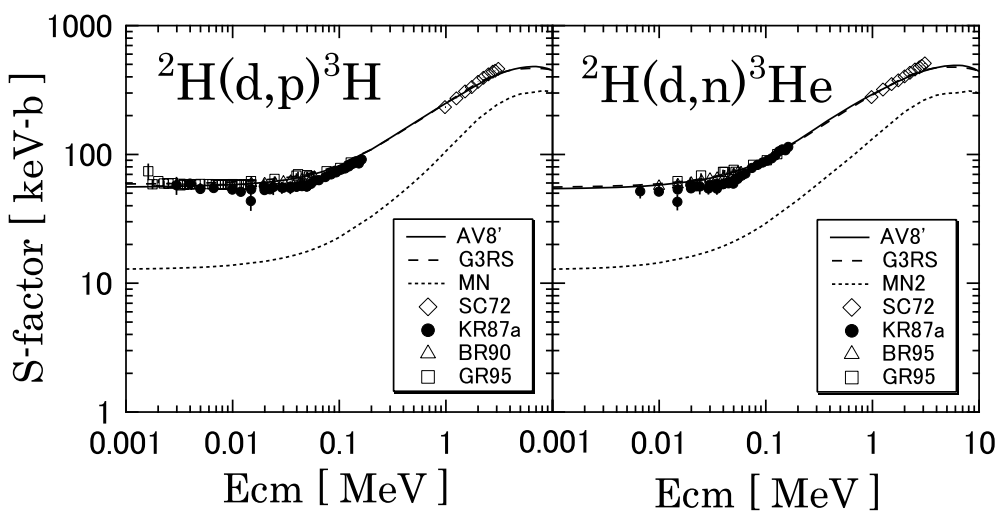

Figure 2: ${ }^{2} \mathrm{H}(d, p){ }^{3} \mathrm{H}$ and ${ }^{2} \mathrm{H}(d, n){ }^{3} \mathrm{He}$ astrophysical $S$-factors calculated with the realistic (AV8', G3RS) and the effective (MN) potentials (taken from Ref. [19]). See Ref. [34] for the experimental data.

The ${ }^{2} \mathrm{H}(d, p)^{3} \mathrm{H}$ and ${ }^{2} \mathrm{H}(d, n)^{3} \mathrm{He}$ reactions play an important role in BibBang nucleosynthesis. As the observed $D / H$ ratio is currently known with $1.5 \%$ accuracy, a high precision is required for the reaction rates. The cross sections have been measured by several groups $[35,36]$, but the extrapolations down to low energies are still uncertain. A compilation of the latest data has been undertaken to reduce the extrapolation uncertainties [37]. In order to disentangle among the different data sets, the ratio $(d, n) /(d, p)$ provides a stronger constrain than the individual cross sections. At low energies, this ratio essentially depends on the Coulomb interaction, and is therefore expected to be very reliable in an ab initio calculation. This ratio is shown in Fig. 3, with values deduced from recent experiments.

As expected this ratio is close to unity since the entrance channel is 


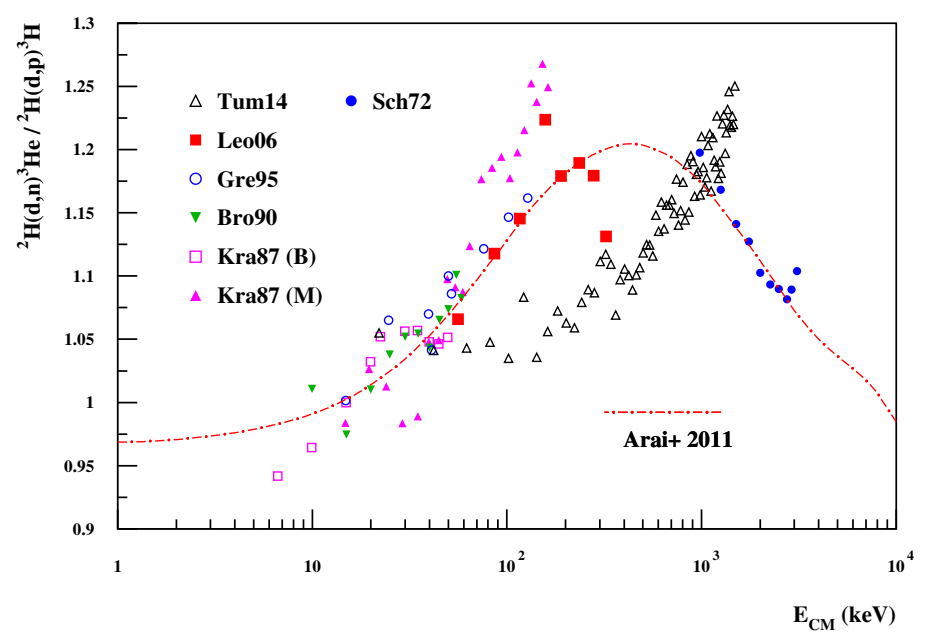

Figure 3: Ratios of ${ }^{2} \mathrm{H}(d, n)^{3} \mathrm{He}$ to ${ }^{2} \mathrm{H}(d, p)^{3} \mathrm{H}$ experimental and theoretical [19] $S$-factors.

common to both reactions. The ab initio calculation predicts a smooth increase, with a maximum near $0.6 \mathrm{MeV}$. This behaviour is supported by previous experimental data, in particular by the data of Ref. [35]. However the indirect data of Ref. [36], obtained in the framework of the Trojan Horse method, are probably affected by a systematic error, which shifts this ratio to higher energies.

\section{Conclusion}

Nuclear astrophysics is a broad field, where many nuclear inputs are necessary. In particular, charged-particle cross sections are quite important, and difficult to measure, owing to the low energies and cross sections. Cluster models are well adapted to these reactions, since in the low-mass region, the number of open channels is fairly small. The assumption of a cluster structure is in general realistic and allows to find approximate solutions of then $A$-body Schrödinger equation. Many applications have been performed so far in nuclear astrophysics. One of the future challenges for these models is the use of more realistic nucleon-nucleon interactions, and their extension to higher mass systems.

We have discussed different theoretical models often used in nuclear astrophysics. The potential model and the $R$-matrix method are widely ap- 
plied in this field; they are fairly simple and well adapted to low-energy reactions. On the other hand microscopic cluster models have a stronger predictive power, since the only rely on a nucleon-nucleon interaction, and on the assumption of a cluster structure for the nucleus. An analysis of the ${ }^{2} \mathrm{H}(d, n)^{3} \mathrm{He}$ and ${ }^{2} \mathrm{H}(d, p)^{3} \mathrm{H}$ cross sections suggests that the Trojan Horse data of Tumino et al. [36] are probably affected by a systematic error.

\section{References}

[1] C. Iliadis, Nuclear Physics of Stars (Wiley-VCH Verlag GmbH, 2007)

[2] P. Descouvemont, M.S. Hussein, A. Lépine-Szily, Nuclear reactions in stars: theoretical and experimental aspects (Nova Science, New-York, 2013)

[3] P. Descouvemont, Theoretical models for nuclear astrophysics (Nova Science, New York, 2003)

[4] P. Descouvemont, T. Rauscher, Nucl. Phys. A 777, 137 (2006)

[5] A.M. Lane, R.G. Thomas, Rev. Mod. Phys. 30, 257 (1958)

[6] P. Descouvemont, D. Baye, Rep. Prog. Phys. 73, 036301 (2010)

[7] T.A. Tombrello, Nucl. Phys. 71, 459 (1965)

[8] I. Thompson, F. Nunes, Nuclear Reactions for Astrophysics: Principles, Calculation and Applications of Low-Energy Reactions (Cambridge University Press, 2009)

[9] K. Wildermuth, Y.C. Tang, A Unified Theory of the Nucleus (Vieweg, Braunschweig, 1977)

[10] W. Hauser, H. Feshbach, Phys. Rev. 87, 366 (1952)

[11] C. Bertulani, Comput. Phys. Commun. 156, 123 (2003)

[12] C. Angulo, P. Descouvemont, Phys. Rev. C 61, 064611 (2000)

[13] C.R. Brune, Phys. Rev. C 66, 044611 (2002)

[14] A. Kievsky et al., J. Phys. G 35, 063101 (2008)

[15] P. Navrátil, R. Roth, S. Quaglioni, Phys. Rev. C 82, 034609 (2010) 
[16] E. Caurier, P. Navrátil, Phys. Rev. C 73, 021302 (2006)

[17] T. Neff, Phys. Rev. Lett. 106, 042502 (2011)

[18] P. Navrátil, S. Quaglioni, Phys. Rev. C 83, 044609 (2011)

[19] K. Arai, S. Aoyama, Y. Suzuki, P. Descouvemont, D. Baye, Phys. Rev. Lett. 107, 132502 (2011)

[20] P. Descouvemont, M. Dufour, Clusters in Nuclei, Vol. 2 (Springer, 2012)

[21] H. Horiuchi, Prog. Theor. Phys. Suppl. 62, 90 (1977)

[22] A.B. Volkov, Nucl. Phys. 74, 33 (1965)

[23] D.R. Thompson, M. LeMere, Y.C. Tang, Nucl. Phys. A 286, 53 (1977)

[24] M. Dufour, P. Descouvemont, Phys. Lett. B 696, 237 (2011)

[25] Q.K.K. Liu, H. Kanada, Y.C. Tang, Phys. Rev. C 23, 645 (1981)

[26] H.M. Hofmann, G.M. Hale, Nucl. Phys. A 613, 69 (1997)

[27] P. Descouvemont, D. Baye, Nucl. Phys. A 573, 28 (1994)

[28] P. Descouvemont, Nucl. Phys. A 596, 285 (1996)

[29] D. Baye, M. Kruglanski, Phys. Rev. C 45, 1321 (1992)

[30] K. Sabourov et al., Phys. Rev. C 70, 064601 (2004)

[31] K. Arai, S. Aoyama, Y. Suzuki, Phys. Rev. C 81, 037301 (2010)

[32] B.S. Pudliner et al., Phys. Rev. C 56, 1720 (1997)

[33] R. Tamagaki, Prog. Theor. Phys. 39, 91 (1968)

[34] C. Angulo et al., Nucl. Phys. A 656, 3 (1999)

[35] D.S. Leonard et al., Phys. Rev. C 73, 045801 (2006)

[36] A. Tumino et al., Astrophys. J. 785, 96 (2014)

[37] A. Coc et al., to be published (2015) 